\title{
How the Size of a Coalition Affects Its Chances to Influence an Election
}

\author{
Arkadii Slinko \\ Department of Mathematics, University of Auckland, Auckland, New Zealand, e-mail: \\ a.slinko@auckland.ac.nz
}

Received: date / Revised version: date

Summary: Since voting rules are prototypes for many aggregation procedures, they also illuminate problems faced by economics and decision sciences. In this paper we are trying to answer the question: How large should a coalition be to have a chance to influence an election? We answer this question for all scoring rules and multistage elimination rules, under the Impartial Anonymous Culture assumption. We show that, when the number of participating agents $n$ tends to infinity, the ratio of voting situations that can be influenced by a coalition of $k$ voters to all voting situations is no greater than $D_{m} \frac{k}{n}$, where $D_{m}$ is a constant which depends only on the number $m$ of alternatives but not on $k$ and $n$. Recent results on individual manipulability in three alternative elections show that this estimate is exact for $k=1$ and $m=3$.

Key words: Social Choice Rule, Impartial Anonymous Culture, Coalition, Manipulability

\section{JEL Classification Numbers: D7}

\section{Introduction}

The well-known impossibility theorem of Gibbard and Satterthwaite $[7,18]$ states that every non-dictatorial social choice function at certain profiles is manipulable by a single individual. Despite the negativity of this result, some hope remained that for sufficiently large societies this phenomenon might be rare. Pattanaik [12] conjectured that "the possibility of strategic voting by single individuals will be smaller the greater the total number of the individuals.” Three years later Pattanaik 
([13], p.187) commented that "if the likelihood of such strategic voting is negligible, then one need not be unduly worried about the existence of the possibility as such."

And although Nitzan [11] experimentally showed the severity of the manipulation phenomenon for societies with small number of voters, Pattanaik's conjecture was confirmed on many occasions. Pazner and Wesley [14], Peleg [15], Fristrup and Keiding [5] showed that for all weighted scoring rules the ratio of manipulable profiles to the total number of profiles tends to zero as the number of voters tends to infinity. Slinko $[19,20]$ showed that, for large societies satisfying the Impartial Culture (IC) assumption, and for all classical social choice rules, the probability of drawing an individually manipulable profile at random is of order $O(1 / \sqrt{n})$ and for the plurality rule it is even $\Theta(1 / \sqrt{n}) .{ }^{1}$

As recent numerical results of Lepelley and Valognes [10] show, possibility of manipulation may crucially depend on the degree of social homogeneity. For a relatively new hypothesis, called the Impartial Anonymous Culture (IAC) assumption (Berg and Lepelley [3] attribute this terminology to Gehrlein), which models societies with a certain degree of social homogeneity, much work was concentrated on the case of three-alternative elections. This hypothesis, as will be explained below, is formulated in terms of voting situations and not profiles and it stipulates that all voting situations are equiprobable. It is remarkable that for the Copeland and the Borda rules the probability of a manipulable voting situation occurring in threealternative elections can be calulated exactly. This was done by Favardin et al. [4], using the technique developed in Huang and Chua [8]. In particular, they showed that, asymptotically, for the Borda rule the probability that a single individual will be able to manipulate is equal to $\frac{25}{12 n}$ and for the Copeland rule this probability is $\frac{5}{4 n}$. This suggests that under the IAC the probability to obtain a manipulable profile converges to zero much faster than under the IC. This occurs because the degree of homogenety of the society under the IAC assumption is greater than for the IC (see [3] for details).

The concept of coalitional manipulability existed in the literature for quite some time (e.g., [2]), and it was studied under several models. Lepelley and Mbih [9] and Favardin et al [4] call a voting situation coalitionally manipulable, if there exists a coalition of any size which can manipulate the election. This concept is closely related to the concept of coalitional instability [9], which, in turn, is equivalent to the Nash-Farquharson-Shubik concept of strong equilibrium in game theory. Saari [17] discussed micro and macro manipulability but in a different model: these roughly correspond to the individual manipulability and coalitional manipulability, as it was defined above.

Lepelley and Mbih [9] and Favardin et al. [4] showed that if we do not restrict the size of the coalition which is allowed to manipulate, then, for $m=3$, the ratio of coalitionally manipulable voting situations to all voting situations does not go to

${ }^{1}$ We write $g(n)=O(f(n))$ in case there is a positive constant $C$ such that $|g(n)| \leq$ $C|f(n)|$ for all sufficiently large values of $n$ and $g(n)=\Theta(f(n))$ if there are positive constants $C_{1}$ and $C_{2}$ such that $C_{1}|f(n)| \leq|g(n)| \leq C_{2}|f(n)|$ for all sufficiently large $n$. 
zero but to a nonzero limit, which is $\frac{7}{24}$ for the plurality rule, $\frac{3}{16}$ for the Copeland rule and approximately $\frac{1}{2}$ for the Borda rule.

Although the concept of coalitional manipulability discussed above is important, it does not enable us to investigate how the probability to manipulate depends on the size of the coalition. To make it possible, the author introduced new concepts of $k$-manipulability and $k$-instability of a profile or a voting situation [21]. A profile or a voting situation is said to be $k$-manipulable, if there exists a coalition of size $k$ or less which is capable of manipulation, and $k$-unstable if some coalition of size $k$ or less can influence the election. Assuming the IC conjecture, Slinko [21] showed that, for any faithful scoring rule, when the number of participating agents $n$ tends to infinity, the probability that a random profile can be influenced by a coalition of size $C n^{\alpha}$, with $0 \leq \alpha<1 / 2$ and $C$ constant, is of order $O\left(1 / n^{1 / 2-\alpha}\right)$. Moreover, Pritchard and Slinko [16] showed that in this case the minimum average size of manipulating coalition is asymptotically equal to $C_{m} \sqrt{n}$, where $C_{m}$ depends on the rule and on $m$ but not on $n$.

In this paper we show that, for the IAC assumption, the probability that a random voting situation can be influenced by a coalition of size $k=C n^{\alpha}$, with $C>0$ and $0 \leq \alpha<1$, is of order $O\left(n^{\alpha-1}\right)$. In particular, for $k=1$, we obtain that the probability that a random voting situation can be influenced by a single individual is of order $O(1 / n)$. On the other hand, as we mentioned above, Favardin et al.,[4] proved that for three-alternative elections the probability that a random voting situation is individually manipulable is of the same order $O(1 / n)$. This leads to a hypothesis that the result of this paper is also exact for $k>1$.

\section{Definitions and Basic Concepts}

Let $A$ and $\mathcal{N}$ be two finite sets, of cardinality $m$ and $n$ respectively. The elements of $A$ will be called alternatives, the elements of $\mathcal{N}$ agents. We assume that the agents have preferences over the set of alternatives. By $\mathcal{L}=\mathcal{L}(A)$ we denote the set of all linear orders on $A$; they represent the preferences of agents over $A$. The cardinality of this set is $M=m$ !, and we list these linear orders in some way $R_{1}, \ldots, R_{M}$ and fix this order.

The well-known Impartial Culture (IC) assumption stipulates that all voters are independent, and that they can choose any linear order on $A$ with equal probability $1 / M$. Unlike the IC, the Impartial Anonymous Culture (IAC) assumption does not operate in terms of individual voters but rather in terms of voting situations that may occur.

Suppose that $n_{i}$ voters chose the linear order $R_{i}$ as their preference over the set of alternatives $A$. Then we say that a voting situation $S=\left(n_{1}, n_{2}, \ldots, n_{M}\right)$ occurred. In other words, a voting situation is a multiset on $\mathcal{L}$ of cardinality $n$. The IAC assumes that all voting situations are equiprobable. Since the total number of voting situations is $\left(\begin{array}{c}n+M-1 \\ n\end{array}\right)$, the probability of each particular voting situation under the IAC is $\left(\begin{array}{c}n+M-1 \\ n\end{array}\right)^{-1}$. 
Hence, unlike the IC, the IAC is a model for the society as a whole and not a model for the behavior of individual voters. For a comprehensive survey of various probability models used in social choice studies see [3].

Let $\mathcal{S}_{n}(A)$ be the set of all voting situations for $n$ voters and the set $A$ of alternatives. Under the IAC, any family of mappings $F=\left\{F_{n}\right\}, n \in \mathbb{N}$,

$$
F_{n}: \mathcal{S}_{n}(A) \rightarrow A,
$$

is called a social choice function (SCF). For historical reasons, SCFs are often called rules.

Definition 1 Let $S$ be a voting situation. We say that a voting situation $S^{\prime}$ occurred as a result of change of mind of $k$ voters, if some $k$ voters who previously submitted linear orders $R_{i_{1}}, \ldots, R_{i_{k}}$ now submit linear orders $R_{j_{1}}, \ldots, R_{j_{k}}$ while the remaining voters submit their original linear orders.

Definition 2 Let $F$ be an SCF and let $S$ be a voting situation. We say that $S$ is $k$-manipulable for $F$ if there is a voting situation $S^{\prime}$, which occurred as a result of change of mind of $k$ voters, with the linear orders $R_{i_{1}}, \ldots, R_{i_{k}}$ being replaced by the linear orders $R_{j_{1}}, \ldots, R_{j_{k}}$, such that $F\left(S^{\prime}\right) R_{i_{s}} F(S)$ for all $s=1,2, \ldots, k$. We also say that a voting situation $S$ is $k$-unstable if there exists a voting situation $S^{\prime}$, which occurs as a result of change of mind of $k$ voters, such that $F\left(S^{\prime}\right) \neq$ $F(S)$.

Every $k$-manipulable voting situation is $k$-unstable, but the reverse is not always true. For $k=1$ we get individual manipulability and individual instability.

The concept of an unstable profile was introduced for the IC assumption by Pazner and Wesley [14] and Peleg [15] and the concept of an unstable voting situation (individual and coalitional) was discussed in Lepelley and Mbih [9]. However, the concept of coalitional manipulability and coalitional instability discussed there does not make any reference to the size of the manipulating coalition.

For our study we will use the following two indices of group manipulability. Given the rule $F$, the index of $k$-manipulability of $F$ under the IAC is

$$
K_{F}(n, m, k)=\frac{d_{F}(n, m, k)}{\left(\begin{array}{c}
n+M-1 \\
n
\end{array}\right)},
$$

where $d_{F}(n, m, k)$ is the total number of all $k$-manipulable voting situations, and the index of instability

$$
L_{F}(n, m, k)=\frac{e_{F}(n, m, k)}{\left(\begin{array}{c}
n+M-1 \\
n
\end{array}\right)},
$$

where $e_{F}(n, m, k)$ is the total number of all $k$-unstable voting situations. We note that under the IAC the set of all voting situations $\mathcal{S}_{n}(A)$ is assumed to be a discrete probability space with the uniform distribution, hence the indices $K_{F}(m, n, k)$ and $L_{F}(m, n, k)$ become the probabilities of drawing at random a $k$ manipulable voting situation, or a $k$-unstable voting situation, respectively. Since 
$K_{F}(m, n, k) \leq L_{F}(m, n, k)$, any upper bound that we can obtain for $L_{F}(n, m, k)$ will be an upper bound for $K_{F}(m, n, k)$.

In this paper we prove that if $F$ is any scoring rule or multistage elimination rule, then

$$
L_{F}(n, m, k) \leq D_{m} \frac{k}{n},
$$

where $D_{m}$ is the constant that depends only on $m$ but not on $k$ and $n$. Therefore, if $n$ and $k$ both tend to infinity so that $k=o(n),{ }^{2}$ then $L_{F}(n, m, k) \rightarrow 0$. This means that any such $F$, asymptotically, cannot be manipulated by coalitions of size $k$.

It is interesting to compare this result with the existing results for the IC. The corresponding indices are defined as follows. The index of $k$-manipulability of $F$ will be

$$
K_{F}(n, m, k)=\frac{d_{F}(n, m, k)}{(m !)^{n}},
$$

where $d_{F}(n, m, k)$ is the total number of all $k$-manipulable profiles, and the index of instability of $F$ will be

$$
L_{F}(n, m, k)=\frac{e_{F}(n, m, k)}{(m !)^{n}},
$$

where $e_{F}(n, m, k)$ is the total number of all $k$-unstable profiles. Peleg [15] proved that if $k=o(\sqrt{n})$, then $L_{F}(n, m, k) \rightarrow 0$. It also follows from Theorem 2 of [20] that for any faithful scoring rule $F$ and any multistage elimination rule based on the scores of $F$

$$
L_{F}(n, m, k) \leq C_{m} \frac{k}{\sqrt{n}},
$$

where $C_{m}$ is a constant which depends only on $m$ but not on $k$ and $n$.

\section{The Main Combinatorial Result}

Definition 3 Let $n, \ell$ be positive integers. Any $\ell$-tuple $\left(n_{1}, n_{2}, \ldots, n_{\ell}\right)$ of nonnegative integers such that

$$
n_{1}+n_{2}+\ldots+n_{\ell}=n
$$

will be called an $\ell$-composition of $n$.

Note that this definition slightly differs from the classical definition of a composition (see, for example, [1]) since the summands in our definition may be zero. In this section $n$ and $\ell$ will be fixed.

\footnotetext{
${ }^{2}$ The notation $g(n)=o(f(n))$ means that $g(n) / f(n) \rightarrow 0$, when $n \rightarrow \infty$.
} 
Definition 4 Let $k_{1}, \ldots, k_{\ell}$, and $p$ be integers. We say that an $\ell$-composition $\left(n_{1}, n_{2}, \ldots, n_{\ell}\right)$ of $n$ satisfies the equation

$$
\sum_{i=1}^{\ell} k_{i} x_{i}=p
$$

iff $\sum_{i=1}^{\ell} k_{i} n_{i}=p$. This equation will be said to be nontrivial if $k_{i} \neq 0$ for some $i$ and not all coefficients $k_{i}$ are equal.

The nontriviality condition is essential. If all coefficients are zero, this clearly does not impose any restriction on $\ell$-composition. If $k_{1}=\ldots=k_{\ell}$ the equation does not impose any restriction either since it is then a multiple of

$$
\sum_{i=1}^{\ell} x_{i}=n
$$

Theorem 1 Let $\ell, n$ be fixed positive integers and let $\mathcal{S}(\ell, n)$ be the set of all possible $\ell$-compositions of $n$. Suppose that the set $\mathcal{S}(\ell, n)$ is given the structure of a discrete probability space with the uniform distribution. Then the probability $P(\ell, n)$ of choosing an $\ell$-composition satisfying a given nontrivial equation is less than or equal to $C_{\ell} / n$, where $C_{\ell}$ is a constant which depends only on $\ell$ but not on $n$.

Proof: Let us fix a nontrivial equation (5). The statement will be proved by induction on $\ell$. For $\ell=2$ the equation (5) becomes

$$
a x_{1}+b x_{2}=c,
$$

where either $a$ or $b$ (or both) are nonzero integers and $a \neq b$. The determinant $\left|\begin{array}{ll}a & b \\ 1 & 1\end{array}\right|=a-b$ is therefore nonzero. Hence we get at most one pair $\left(n_{1}, n_{2}\right)$, satisfying (7). The total number of 2-compositions is therefore no greater than $n+1$, and the probability that a random pair satisfies (7) is less than $1 / n$, as required. We can take $C_{1}=1$, and this gives a basis for the induction.

Suppose now that, for all $k<\ell$, the constants $C_{k}$ exist such that $P(k, n) \leq$ $C_{k} / n$. We will show that for $C_{\ell}=\max \left(l, \frac{\ell^{2} C_{\ell-1}}{\ell-1}\right)$ the inequality $P(\ell, n) \leq$ $C_{\ell} / n$ will also be satisfied. Let us consider the following three cases:

Case 1: Only one coefficient $k_{i}$ in the equation (5) is nonzero. In this case $n_{i}=p / k_{i}$ is fixed and the total number of $\ell$-compositions with the fixed $n_{i}$ can be estimated as follows:

$$
\left(\begin{array}{c}
n-n_{i}+\ell-2 \\
n-n_{i}
\end{array}\right) \leq\left(\begin{array}{c}
n+\ell-2 \\
n
\end{array}\right) \leq \frac{\ell}{n}\left(\begin{array}{c}
n+\ell-1 \\
n
\end{array}\right) .
$$

Since the total number of $\ell$-compositions is $\left(\begin{array}{c}n+\ell-1 \\ n\end{array}\right)$, the probability of this event is not greater than $\ell / n \leq C_{\ell} / n$. 
Case 2: All coefficient in the equation (5) but $k_{i}$ are equal. Suppose $k_{j}=k$ for $j \neq i$. Then, subtracting from (5) the equation (6) $k$ times we will obtain the equation $\left(k_{i}-k\right) x_{i}=p-k n$, which reduces this case to Case 1 .

Case 3: At least two coefficients among the $k_{1}, \ldots, k_{\ell}$ in the equation (5) are nonzero and no $\ell-1$ coefficients are equal. In this case we may assume that for any $\ell$-composition $S=\left(n_{1}, n_{2}, \ldots, n_{\ell}\right)$ of $n$ satisfying (5) and for any $i=$ $1,2, \ldots, \ell$, the $(\ell-1)$-composition

$$
S^{\prime}=\left(n_{1}, \ldots, n_{i-1}, n_{i+1}, \ldots, n_{\ell}\right)
$$

of $n-n_{i}$ satisfies the nontrivial equation

$$
k_{1} x_{1}+\ldots+k_{i-1} x_{i-1}+k_{i+1} x_{i+1}+\ldots+k_{\ell} x_{\ell}=p-k_{i} n_{i} .
$$

Hence we may assume that any $\ell$-composition $S=\left(n_{1}, n_{2}, \ldots, n_{\ell}\right)$ of $n$ satisfying a nontrivial equation (5), will still satisfy a nontrivial equation if we remove any of the $n_{i}$ 's from it.

Let us now estimate $P(\ell, n)$. This estimation will be based on the observation that $n_{i} \leq n / \ell$ for at least one $i \in\{1, \ldots, \ell\}$. Because of that

$$
P(\ell, n) \leq \sum_{i=1}^{\ell} \sum_{k=0}^{n / \ell} \operatorname{Prob}\left\{n_{i}=k\right\} P(\ell-1, n-k) .
$$

Since, for $k \leq n / \ell$, by the induction hypothesis we obtain

$$
P(\ell-1, n-k) \leq \frac{C_{\ell-1}}{n-k} \leq \frac{C_{\ell-1}}{n-n / \ell}=\frac{\ell}{\ell-1} \frac{C_{\ell-1}}{n},
$$

we get

$$
P(\ell, n) \leq \sum_{i=1}^{\ell} \sum_{k=0}^{n / \ell} \operatorname{Prob}\left\{n_{i}=k\right\} P(\ell-1, n-k) \leq \frac{\ell^{2} C_{\ell-1}}{\ell-1} \cdot \frac{1}{n}=\frac{C_{\ell}}{n} .
$$

This proves the theorem.

\section{Asymptotic Strategy-Proofness}

One of the most important classes of SCFs was introduced by Gärdenfors [6]: they are called representable voting functions.

Definition 5 A representation function is a function $f: \mathcal{L}(A) \times A \rightarrow \mathbb{R}$ such that

$$
a R_{i} b \Longrightarrow f\left(R_{i}, a\right) \geq f\left(R_{i}, b\right) .
$$

It is called faithful if

$$
\left(a R_{i} b \text { and } a \neq b\right) \Longrightarrow f\left(R_{i}, a\right)>f\left(R_{i}, b\right) .
$$


Definition 6 A representation function $f: \mathcal{L}(A) \times A \rightarrow \mathbb{R}$ is called positionalist, if $f(R, a)$ depends only on the cardinality of the lower contour set $L(R, a)=\{b \in$ $A \mid a R b$ and $a \neq b\}$.

The simplest positionalist representation function (which is used to define the Borda rule) can be defined by setting $f(R, a)=\operatorname{card}(L(R, a))$. It is easy to see that it is faithful.

Let $f$ be a representation function and $S=\left(n_{1}, n_{2}, \ldots, n_{M}\right) \in \mathcal{S}_{n}(A)$ be a voting situation. We define the score function $S c_{f}: \mathcal{S}_{n}(A) \times A \rightarrow \mathbb{R}$ by

$$
S c_{f}(S, a)=\sum_{i=1}^{M} n_{i} f\left(R_{i}, a\right), \quad a \in A .
$$

Definition 7 An SCF F is called (faithfully) representable if there exists a (faithful) representation function $f$ such that for every $S \in \mathcal{S}_{n}(A)$ we have $F(S)=a_{i}$ if and only if

$$
\begin{aligned}
& j<i \Longrightarrow S c_{f}\left(S, a_{i}\right)>S c_{f}\left(S, a_{j}\right), \\
& j>i \Longrightarrow S c_{f}\left(S, a_{i}\right) \geq S c_{f}\left(S, a_{j}\right) .
\end{aligned}
$$

When $f$ is positionalist, any SCF is also called a (faithful) scoring rule (or pointvoting scheme).

Every scoring rule $F$ with a representation function $f$ is characterised by the respective vector of weights $W_{f}=\left(w_{1}, \ldots, w_{m}\right)$. The weights are chosen so that for any $Q \in \mathcal{L}(A)$ the equation $w_{i}=f(Q, a)$ holds if and only if $\operatorname{card}(L(Q, a))=m-i$. The weights must satisfy the condition

$$
w_{1} \geq w_{2} \geq \ldots \geq w_{m}=0,
$$

and we can consider them to be integers. It is clear that the scoring rule $F$ is faithful iff $w_{i} \neq w_{i+1}$ for all $i=1,2, \ldots, m-1$.

For each voting situation $S \in \mathcal{S}_{n}(A)$ the value of the scoring function $f$ on $a$, which we will simply call the score of $a$, can be now computed as follows. Let $I_{a}=\left(i_{1}, \ldots, i_{m}\right)$ be the vector such that the number $i_{k}$ shows how many times the alternative $a$ was ranked $k$ th. Then

$$
S c_{f}(S, a)=W_{f} \cdot I_{a}=\sum_{\ell=1}^{m} w_{\ell} i_{\ell} .
$$

The most commonly used scores are the plurality score $S c_{P}(S, a)$, when $P$ is the plurality rule defined by the vector of weights $W_{P}=(1,0, \ldots, 0)$, and the Borda score $S c_{B}(S, a)$, where $B$ is the Borda rule defined by the vector of weights $W_{B}=(m-1, m-2, \ldots, 1,0)$. The Approval Voting score with a fixed number $k$ of approvals is given by the vector of weights $W_{A}=(1,1, \ldots, 1,0, \ldots, 0)(k$ ones). When $k=m-1$ it bears the name of the Antiplurality score.

Another related class of social choice rules are multistage elimination rules for which the winner is determined in several stages. At every stage one (or more) 
of the alternatives is eliminated on the basis of a certain "global" information, i.e. the eliminated alternative must be the worst one relative to a certain global characteristic. This characteristic is normally related to the scores of alternatives defined in the previous section.

Among these the best known is the run-off procedure for which at the first stage $m-2$ alternatives with the lowest plurality scores are eliminated. Hare's Rule (or Single Transferable Vote) stipulates that at every stage only one alternative with the minimal plurality score is eliminated. The Inverse Borda rule acts exactly as Hare's rule but the Borda scores are used instead of the plurality scores. Coombs' procedure eliminates the alternative with the lowest Antiplurality score. Nanson's procedure eliminates all alternatives whose Borda scores are lower than the average Borda score. Clearly, for each of these rules, given an unstable voting situation certain scores for some two alternatives must be close, and it is easy to specify how close.

The following obvious lemma explains how the scores can be changed during a manipulation attempt.

Lemma 1 Let $F$ be any scoring rule with a representation function $f$ characterised by the vector of weights $W_{f}=\left(w_{1}, \ldots, w_{m}\right)$ satisfying (12). Let $S$ be a voting situation and let $S^{\prime}$ be another voting situation which occurred as a result of change of mind of $k$ voters. Then

$$
\left|S c_{f}(S, a)-S c_{f}\left(S^{\prime}, a\right)\right| \leq k w_{1} .
$$

Proof Straightforward.

Theorem 2 For any scoring rule or multistage elimination rule F, under the IAC, there exists a constant $D_{m}$, which depends only on $m$ but not on $n$ and $k$, such that

$$
L_{F}(m, n, k) \leq D_{m} \frac{k}{n}
$$

Proof Let $f$ be the representation function of the scoring rule which is used either directly or provides the score function for elimination of alternatives. Let $w_{1}$ be the senior weight of the corresponding vector of integer-valued weights $W_{f}$.

We will prove that the inequality (13) will be satisfied for the constant $D_{m}=$ $\left(4 w_{1}+1\right) C_{M}$, where $C_{M}$ is the constant from Theorem 1 and $M=m$ !. Due to Lemma 1 a voting situation $S=\left(n_{1}, n_{2}, \ldots, n_{M}\right)$, where $M=m$ !, may be $k$-unstable if for some two alternatives $a$ and $b$

$$
\left|S c_{f}(S, a)-S c_{f}(S, b)\right| \leq 2 k w_{1}
$$

Indeed, suppose that the score of $a$ is greater than that of $b$. From Lemma 1 we see that it may be possible that by switching to a new voting situation, $S^{\prime}$ which occurred as a result of change of mind of $k$ voters, the score of $a$ will be decreased by $k w_{1}$ and simultaneously the score of $b$ will be increased by the same amount. If $a$ had the highest score this may result in the change of the winner. 
The inequality (14), in turn, means that $S$ satisfies one of the following nontrivial equations:

$$
\sum_{i=1}^{M}\left(f\left(R_{i}, a\right)-f\left(R_{i}, b\right)\right) x_{i}=p
$$

$a, b \in A,-2 k w_{1} \leq p \leq 2 k w_{1}$. We have $4 k w_{1}+1$ such equations. Hence by Theorem 1

$$
L_{F}(m, n, k) \leq\left(4 k w_{1}+1\right) \frac{C_{M}}{n} \leq D_{m} \frac{k}{n}
$$

and the theorem is proved.

Corollary 1 For $k=o(n)$ any scoring rule or multistage elimination rule $F$, under the IAC, is asymptotically $k$-coalitionally stable, i.e.,

$$
L_{F}(m, n, k) \rightarrow 0,
$$

when $n \rightarrow \infty$.

Corollary 2 Let $F$ be any scoring rule or multistage elimination rule under the IAC. Suppose that $k \sim n^{\alpha}$, where $0 \leq \alpha<1$. Then the probability that a random voting situation is manipulable for $F$ by a coalition of size $k$ is the order of $O\left(n^{\alpha-1}\right)$. In particular, a random voting situation is manipulable by a single individual with probability which is the order of $O(1 / n)$.

\section{Concluding Remarks}

Comparing the results of this paper with the results obtained under the IC assumption, we observe that the homogenity of the population reduces the possibility of manipulation. Thus, for the IC assumption, when all $n$ voters are totally independent, it follows from [16] that a coalition of the size $C \sqrt{n}$ has a nonzero limiting probability to manipulate an election. In this paper we see that for the IAC assumption, this coalition must be much bigger and must include a fraction of the society. The reason is that the greater homogeneity of the IAC reduces the probability of a close election.

Our analysis suggests that the concepts of $k$-manipulability and $k$-instability introduced in [21] is a useful tool in analysing the impact of the coalition size on the probability to manipulate or to influence an election.

\section{Acknowledgements}

The author is grateful to two anonymous referees whose thoughtful comments and suggestions helped to improve the exposition. 


\section{References}

1. Andrews GE (1976) The theory of partitions. Addison-Wesley, Massachusetts

2. Barbera S (1978) A Note on Group Strategy-Proof Decision Schemes. Econometrica 47: 637-640.

3. Berg S and D Lepelley (1994) On Probability models in voting theory. Statistica Neerlandica 48: 133-146.

4. Favardin P, Lepelley D and J Serais (2002) Borda rule, Copeland method and strategic manipulation. Review of Economic Design 7: 213 -228.

5. Fristrup P and H Keiding (1989) A Note on asymptotical strategy proofness. Economics Letters 31: 307-312.

6. Gärdenfors P (1973) Positionalist voting functions, Theory and Decision 4: 1-24.

7. Gibbard A (1973) Manipulation of voting schemes: A general result. Econometrica 41: $587-601$.

8. Huang HC and VCH Chua (2000) Analytical representation of probabilities under the IAC condition. Social Choice and Welfare 17: 143-156.

9. Lepelley D and B Mbih (1987) The proportion of coalitionally unstable situations under the plurality rule. Economic Letters 24: 311-315.

10. Lepelley D and F Valognes (2002) Voting rules, manipulability and social homogeneity. Public Choice, to appear.

11. Nitzan S (1985) The vulnerability of point-voting schemes to preference variation and strategic manipulation. Public Choice 47: 349-370.

12. Pattanaik PK (1975) Strategic voting without collusion under binary and democratic group decision rules. The Review of Economic Studies 42: 93-103.

13. Pattanaik PK (1978) Strategy and group choice. North-Holland, Amsterdam

14. Pazner EA and E Wesley (1978) Cheatproofness properties of the plurality rule in large societies. The Review of Economic Studies 45: 85-91.

15. Peleg B (1979) A note on manipulability of large voting schemes. Theory and Decision 11: 401-412.

16. Pritchard G and A Slinko (2003) On the Average Minimum Size of Manipulating Coalition, Report Series N. 507. Department of Mathematics. The University of Auckland (http://www.math.auckland.ac.nz/deptdb/dept_reports/507.pdf).

17. Saari D (1990) Susceptibility to manipulation. Public Choice 64: 21-41.

18. Satterthwaite MA (1975) Strategy-proofness and Arrow's conditions: existence and correspondence theorems for voting procedures and social welfare functions. Journal of Economic Theory 10: 187-217.

19. Slinko A (2002) Asymptotic strategy-proofness of the plurality and the run-off rules. Social Choice and Welfare 19: 313-324.

20. Slinko A (2002) On asymptotic strategy-proofness of classical social choice rules. Theory and Decision 52: 389-398.

21. Slinko A (2004) How Large Should a Coalition Be to Manipulate an Election? Mathematical Social Sciences 47: 289-293. 